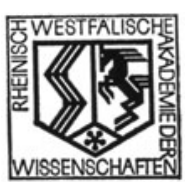


Rheinisch-Westfälische Akademie der Wissenschaften

Natur-, Ingenieur- und Wirtschaftswissenschaften

Vorträge $\cdot$ N 235

Herausgegeben von der

Rheinisch-Westfälischen Akademie der Wissenschaften 


\section{BERNHARD SANN}

Die Senkung der Maschinenleistung bei Steigerung der Gewinnungsleistung und die Einsteuerung von Maschinen für die schälende Gewinnung von Steinkohle

\section{LOTHAR FREYTAG}

Möglichkeiten der Verwirklichung von Forschungsund Versuchsergebnissen in der Konstruktion von Maschinen für die schälende Kohlengewinnung

(W)

Westdeutscher Verlag · Opladen 
216. Sitzung am 6. Juni 1973 in Düsseldorf

ISBN 978-3-663-01773-8 ISBN 978-3-663-01772-1 (eBook)

DOI 10.1007/978-3-663-01772-1

(c) 1973 by Westdeutscher Verlag GmbH Opladen

Gesamtherstellung: Westdeutscher Verlag GmbH 


\section{Inhalt}

Bernhard Sann, Aachen

Die Senkung der Maschinenleistung bei Steigerung der Gewinnungsleistung und die Einsteuerung von Maschinen für die schälende Gewinnung von Steinkohle ....................... 7

Die Maschinen- und Gewinnungsleistung des Hobels ........... 9

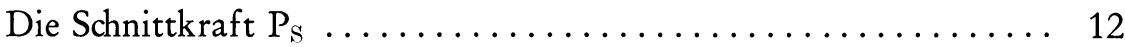

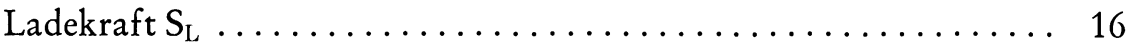

Die Reibungskraft $\mathrm{W}_{\mathrm{H}}$ des Hobels, bedingt durch das Eigengewicht und die zur Führung des Hobels erforderlichen Querabstützkräfte . . 18

Die Reibungskraft $W_{K}$ der Kette, bedingt durch ihr Eigengewicht . . 22

Die Reibungskraft $W_{\mathrm{EK}}$ der Kette, bedingt durch Abwinkelungen der Kettenführungsbahn $\ldots \ldots \ldots \ldots \ldots \ldots \ldots \ldots \ldots \ldots \ldots \ldots \ldots$

Die Verwendung rollender Reibung $\ldots \ldots \ldots \ldots \ldots \ldots \ldots \ldots \ldots \ldots$

Maschinentechnische Verkürzung der Streblänge .......... 30

Die Einsteuerung des Hobels in den Flözhorizont ........... 30

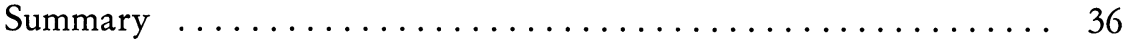

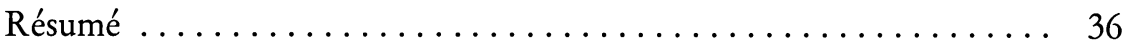

Lothar Freytag, Westfalia Lünen

Möglichkeiten der Verwirklichung von Forschungs- und Versuchsergebnissen in der Konstruktion von Maschinen für die schälende Kohlengewinnung $\ldots \ldots \ldots \ldots \ldots \ldots \ldots \ldots \ldots \ldots \ldots \ldots \ldots$

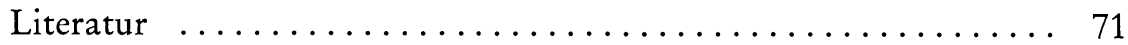

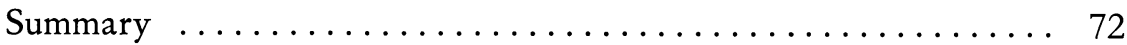

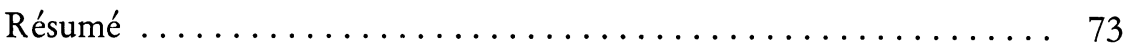


Diskussionsbeiträge

Professor Dr. phil. Martin Schmeißer; Bergassessor Lothar Freytag; Professor Dr.-Ing. Bernhard Sann; Professor Dr.-Ing. Volker Aschoff; Professor Dr. rer. nat. Rudolf Schulten; Regierungsdirektor i. R. Dipl.-Ing. Kurt Boebmer; Professor Dr.-Ing., Dr.-Ing. E. h., Dr. sc. a. h. c. Hermann Schenck; Professor Dr.-Ing. August Wilhelm

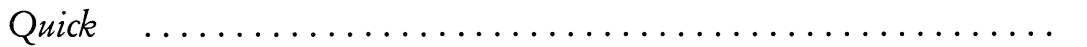

